\title{
Adaptive strip sampling in forest inventory of scattered species of Ulmus glabra in Hyrcanian Forests, northern Iran
}

\author{
MARYAM FAZLOLLAHI MOHAMMADI", FATEMEH AHMADLO, SAHAR MOKARAM KESHTIBAN \\ Department of Forestry, Faculty of Natural Resources and Marine Sciences, Tarbiat Modares University, 46417-76489, Noor, Mazandaran, Iran, Tel.: \\ +98-122-6253101 (-3), Fax.: +98-122-6253499. `email: mfazlollahi83@yahoo.com
}

Manuscript received: 8 January 2015. Revision accepted: 30 March 2015.

\begin{abstract}
Fazlollahi Mohammadi M, Ahmadlo F, Keshtiban SM. 2015. Adaptive strip sampling in forest inventory of scattered species of Ulmus glabra in Hyrcanian Forests, northern Iran. Nusantara Bioscience 7: 48-54. Some populations of tree species may be sparse, but they are highly geographically clustered. A simple random sample of plots could then yield mainly empty plots. Adaptive Cluster Sampling (ACS) is an efficient design for tallying of these populations. Presented study is designed to estimate the abundance of Wych elm (Ulmus glabra) as a scattered tree species in mountain forests of Iran. This study looks at design-based methods for a forest inventory survey, intending to improve estimation when the target species has a patchy spatial distribution. In particular, we examined the efficiency and feasibility of modified adaptive cluster sampling in combination with strip sampling (SACS) as a new sampling design in Hyrcanian forests, Iran for scattered species. To collect data, we recorded a number of 845 trees to represent locations of individuals in a spatially aggregated population by using GPS 60CX model. In overall, results showed that SACS was underestimated in estimating coefficient of variance $(\mathrm{CV} \mu)$ and $(\mathrm{Var} \mu)$ while it was overestimated in estimating mean of tree density at all quadrate size except for size $15 \mathrm{~m}$ square in compared to conventional strip sampling (CSS). Results evidenced that HH estimator was more efficient than HT ones in estimating mean, $(\mathrm{CV} \mu)$ and variance (Var $\mu$ ) in compared to (CSS) approach. Practically results of our study using SACS confirmed that a $15 \mathrm{~m}$ square sample size of quadrate with four adjacent units was optimized network size to sampling of such populations. Future studies using SACS may be required, especially, in combine to two-phase sampling strategies when cover of these species is of concern.
\end{abstract}

Keywords: Conventional strip inventory, Hyrcanian forests, forest sampling, ratio efficiency, SACS, wych elm

\section{INTRODUCTION}

In ecological studies, efficiently sampling of rare species due to its special geographically spatial distribution pattern is a difficult challenge. Conventional sampling designs may require substantial effort in order to achieve even moderate precision (Evans and Viengkham 2006). A few techniques of sampling designs and response designs with proven track records in forestry are also successfully being employed in ecological surveys. While a cluster pattern plants with heterogeneous spatial distributions (Greig-Smith 1983; Goslee 2006) have been devoted a little attention in ecological studies. In Hyrcanian forests, there are some species such as Wych elm, mountain Ash, Silver lime and Sour cherry, which sometimes have cluster distribution pattern in their site (Sabeti 1976). The distribution of these less common species, spatially comes from some ecological factors such as elevation, light, water, and soil condition, that occur unevenly within forest stands (Barbier 2008), due to within-stand heterogeneity in structure, composition, and abiotic conditions.

Selection of sampling designs must be based on the spatial structure and heterogeneity of the overall plant community or management concern (Stohlgren 2007). For such situations, Thompson (1990) recommended a technique as adaptive cluster sampling (ACS) which sample points are selected according to a sampling design, for example, random or systematic (Acharya et al. 2000), strip and transect (Thompson 1990) sampling, and at each sample location as a quadrant (Stohlgren et al. 1995; Abella and Covington 2004), called initial unit, is laid out. According to Brown (1996), adaptive cluster sampling (ACS) design is efficient only for very patchy populations, but it can be highly inefficient for other less aggregate populations. Cluster sampling is often carried out for achieving convenience or practicality rather than obtaining the lowest variance for a given number of units observed (Thompson 1999). Conners and Schwager (2002), Thompson and Seber (1996) and Christman (1997) found that adaptive cluster sampling for spatially patchy species gave better results than the traditional cluster sampling techniques. If an initial unit of a specific condition is satisfied for example: "at least one target object found or a specific predefined diameter" then the nearest quadrant or units are also sampled if one of these adjacent units meets the condition and so on. That is, the initial unit is step by step enlarged by adding more and more units and becomes an irregularly shaped cluster of sub-units. The vivid advantage of cluster sampling is that it is usually cost less to sample a collection of units in a cluster than to sample an equal number of units selected randomly from the population. ACS designs performed better than the traditional designs for all stocks with small-scale spatial correlation in tree density, yielding estimates with lower variance. ACS estimators were not skewed and had a lower frequency of large errors differences between traditional 
and ACS designs were consistent over multiple realizations of each spatial covariance model. Meanwhile, it is a good potential for situations where standard cluster sampling is ineffective; when the target stock tends to concentrate in a few dense clusters rather than being evenly distributed over the study area (Conners and Schwager 2002).

In this case study, one of the novel techniques sampling design based on adaptive cluster sampling which recently developed is transect-base in forest inventory (Abrahamson et al. 2011). In strip transect random sampling It is assumed that the detectability in the narrow strip is perfect, i.e., every individual in the strip is detected with probability 1 . Individuals detected outside the narrow strip are ignored, because the detectability of these individuals is $<1$. The sampling units generally have different (surface) areas and therefore the density is estimated via the total number of individuals. In order to overcome this drawback, transect sampling along with ACS was applied as a relatively cheap method of estimating objects with rare distribution. Limgta and Fries (1995) concluded that for sparse objects tallied transect-base methods like strip surveying should be more cost-efficient because a larger area is covered. Abrahamson et al. (2011) studied the efficiency of SACS along with the conventional (modified Whittaker plots and Daubenmire and point-line intercept transects) approaches to study understorey sample plants in western Montana, USA and found out that SACS was the only design that efficiently sampled less common species, requiring only $6-11 \%$ as many replicates relative to conventional designs. Ensign et al. (1995) and Barlow (1995) compared line and strip transect methods for estimating fish population and concluded that line transect methods performed well, while, Barlow (1995) who compared strip and line transects to estimate the abundance of cetaceans and found both methods worked well. Salehi and Seber (1997) gave a method for designing an adaptive cluster sample using twophase sampling and concluded that this procedure was effective in comparison with the other sampling techniques, and could be used as a bias reduction technique. The use of such models hinges on being able to satisfactorily model the probability of detecting the object as a function of perpendicular distance $y$, the so-called detection function" $\mathrm{g}(\mathrm{y})$.

Our literature reviews showed that there are a lot of researches that has focused on application of sampling techniques on estimating of abundance and density of understorey and overstorey plants in field, while efficiency of sampling method on responses to disturbance and management of rare species has been received little attention. Therefore, to broaden current knowledge of rare tree species assessment, our study includes the following objective: comparing performance of SACS and conventional strip sampling approaches in an inventory of Wych elm as a scattered tree species in mountainous forests of Iran.

\section{MATERIALS AND METHODS}

\section{Study site}

Present study was carried out at Education and Experimental Forest of Tehran University, located in a temperate forest in the north of Iran between $40^{\circ} 46^{\prime} 91^{\prime \prime} \mathrm{N}$

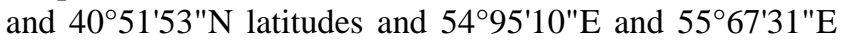
longitudes (Figure 1). Average annual rainfall recorded at the closest national weather station is $918 \mathrm{~mm}$. research area are dominated by Fagus orientalis Lipskey and Carpinus betulus with Acer spp., Alnus spp., Tilia spp., and Ulmus glabra as dominant companion species in understorey. This research was limited into 2 of 7 districts of this forest which includes inclusively Wych elm species more than others.
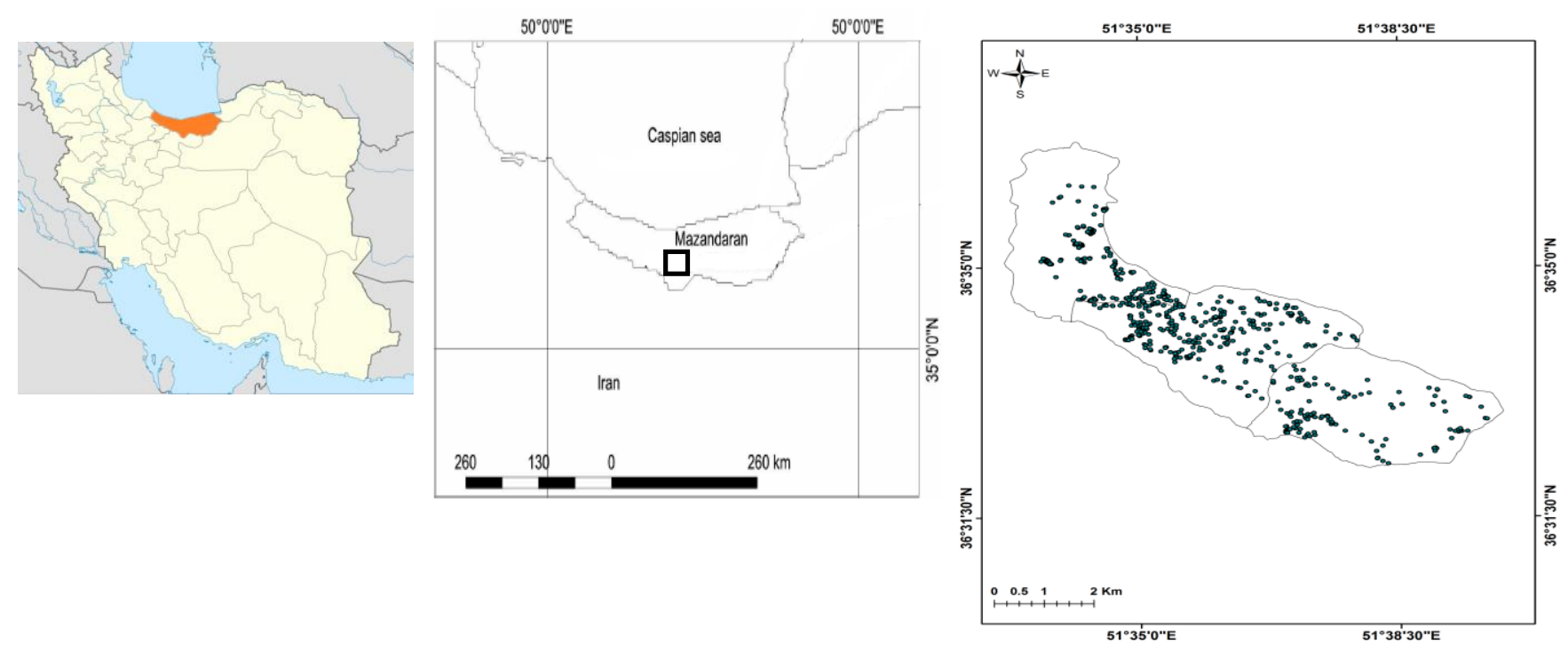

Figure 1. General illustration of SACS approach in the study area 


\section{Data collection}

During June 2012, at each of two sites, we recorded individual target tree species. The $X, Y$ coordinates used for all individuals in the populations are accurate to at least $0.5 \mathrm{~m}$ and were supplied by GPS $60 \mathrm{CX}$ brand. Then, the point data for each tree were imported into ArcGIS 9.3 version, where grid-based sampling universes were simulated comprised of long strip primary units of fixed distance 15, 20 and $25 \mathrm{~m}$ from the centerline of transect. Prior to grid placement, a constant value of 0.01 was added to the $X, Y$ coordinates of the individuals in population.

In current study, transect-based method is accomplished in two steps. In the first step, transect locations are selected according to simple strip design (SSD); each transect then includes a set of secondary sampling units in which the tree density is measured by using number of individual tree with in a fixed distance from the centerline of transect. The type of ACS sampling that applicable to such exercises is, therefore, ACS with primary and secondary units (Thompson 1990; Thompson and Seber 1996; Pontius 1997). We preliminarily designed transect as primary units (continues transect of $15 \times 15,20 \times 20$ and $25 \times 25$ m quadrates) as the first step of SACS and the integrated sampling units were taken into account as secondary units in this form of Strip Adaptive Cluster Sampling (SACS) (Figure 1).

Probability proportional to size was applied to allocate number of initial unit to each district. The length of transect line was equal to the projection of the area onto the line. The initial sampling design consists number of $17(n=17)$ randomly placed, transects over each district (Brown 1999). All of the sampling units/or quadrates in the initial design are measured and the nearest units close to intersected primary unit that meet a pre-specified criterion are identified. Our defined criterion was the presence of a rare species or a Wych elm. For the adaptive stage of sampling secondary units are added in the neighborhood of any secondary unit meeting the ACS condition. The final sample includes the entire initial transects plus, where any transect intersects a high-density cluster, a "cloud" of adaptively added secondary units. The number of units added to the sample depends on the ACS defined condition and neighborhood definition used, as well as on the scale of secondary units and the spatial distribution of the target species. The neighborhood can be defined in several ways, for example, four surrounding quadrants in the neighborhood has value as the critical value; the neighborhood of that quadrat is sampled, and so on. In our study, a neighborhood was defined as four or more adjacent units, which includes more or equal to three observations in itself and sharing a common boundary with the target quadrat. If an additional unit did not meet the criterion of adaptively, would include edge units, were considered networks of size one. The criterion of adaptive added units was that the number of observations in a unit should be greater than or equal to three (Dryer 1999). The group of adjacent quadrates whose values are all at least as great as the critical value was known as a network. The most commonly used scheme is adaptive cluster sampling which takes an initial random sample and then successively adds neighborhoods of units which satisfy a certain criterion, thus building up cluster.

After illustrating the primary unit and developing its neighborhoods, we utilized two common estimators for estimating the total mean density and variance of SACS design. Both estimators balance the total number of trees in a network $\left(y_{k}^{*}\right)$ against the probability of detecting that network, based on its "width" $\left(\mathrm{x}_{\mathrm{k}}\right)$ relative to the initial sampling design. Estimation of the mean over the study area is based on the means within the sampled networks, including a large number of one size networks and few larger networks. The first estimator, which was referred to Hansen-Hurwitz-type (HH), is based on sampling with replacement and draw-by-draw selection probability for each transect. Variance estimation for the $\mathrm{HH}$ estimator is based on variance between densities estimated by each transect. The second estimator, the Horvitz-Thompson-type (HT), may be used when sampling with or without replacement.

Sampling and estimation for the conventional designs were performed using simplest version of Line-Transect Random Sampling in which only the detected individuals are used in the estimation in a narrow strip along the line. This is referred to the narrow-strip method (Gruijter et al. 2005). This method included the number of $17(n=17)$ systematic transects with a random start point to cover study area partially.

\section{Estimators}

\section{Horvitz and Thomson estimator}

$$
\pi_{i}=1-\left[\left(\begin{array}{l}
N-m_{i}-a_{i} \\
n_{1}
\end{array}\right) /\left(\begin{array}{l}
N \\
n_{1}
\end{array}\right)\right]
$$

The $m_{i}$ is known for the units in the sample, but some of the $a_{i}$ are known, this requires sampling around the "empty" sampling units which we do not do. To work around this problem, since we need a probability of selection, we compute the "partial" inclusion probability

$$
\pi_{i}^{\prime}=1-\left[\left(\begin{array}{l}
N-m_{i} \\
n_{1}
\end{array}\right) /\left(\begin{array}{l}
N \\
n_{1}
\end{array}\right)\right]
$$

Which only uses known information and is the probability that the initial sample intersects $A_{i}$. Observations that do not satisfy the condition $C$ are ignored if they are not included in the initial sample. An unbiased estimator for the population mean is then given by

$$
\begin{aligned}
& \hat{\mu}=\frac{1}{N} \sum_{i=1}^{N} \frac{y_{i} I_{i}}{\pi_{i}} \\
& \mu=\text { Total of } y \text { values in the } i^{t h} \text { network. }
\end{aligned}
$$

$I_{i}=1=$ if any quadrant in the $i^{\text {th }}$ network, $0=$ otherwise. This can be rewritten in terms of the distinct networks as; 


$$
\hat{\mu}=\frac{1}{N} \sum_{k=1}^{k} \frac{y_{k}^{*} J_{k}}{\alpha_{k}}=\frac{1}{N} \sum_{k=1}^{k} \frac{y_{k}^{*}}{\alpha_{k}}
$$

Where,

$y_{k}{ }^{*}$ is the sum of the $y_{k}$ values for the network, $K$ is the total number of distinct networks

in the population, $K$ is the number of distinct networks in the sample, and $J_{k}$ is another

indicator function that is 1 if the initial sample intersects the $k_{t h}$ network and 0 otherwise (Tompson 1990).

If there are $x_{k}$ sampling units in the $k_{t h}$ network, then:

$a_{k}=1-\left[\left(\begin{array}{l}N-x_{k} \\ n_{1}\end{array}\right) /\left(\begin{array}{l}N \\ n_{1}\end{array}\right)\right]$

Also, when $x_{k}=1$, then $a_{k}=n_{1} / N$. Further, define $p_{j k}$ to be the probability that the $j_{t h}$ and $k_{t h}$ networks are not intersected, then (Tompson 1992):

$$
p_{j k}=\left(\begin{array}{l}
N-x_{j}-x_{k} \\
n_{1}
\end{array}\right) /\left(\begin{array}{l}
N \\
n_{1}
\end{array}\right)
$$

So that the joint probability that networks $j$ and $k$ are both intersected is:

$a_{j k}=a_{j}+a_{k}+\left(1-\left\{\left(\begin{array}{l}N-x_{j} \\ n_{1}\end{array}\right)+\left(\begin{array}{l}N-x_{k} \\ n_{1}\end{array}\right)-\left(\begin{array}{l}N-x_{j}-x_{k} \\ n_{1}\end{array}\right)\right\},\left(\begin{array}{l}N \\ n_{1}\end{array}\right)\right.$

Now the variance can be derived as:

$$
\operatorname{Var}(\hat{\mu})=\frac{1}{N^{2}}\left[\sum_{j=1}^{k} \sum_{k=1}^{k} \frac{y_{j}^{*} y_{k}^{*}}{a_{j k}}\left(\frac{a_{j k}}{a_{j} a_{k}}-1\right)\right]
$$

Where $a_{j k \equiv} a_{j}$

\section{Hansen-Hurwitz estimator}

$$
\hat{\mu}=\frac{1}{n_{1}} \sum_{i=1}^{N} \frac{y_{i} f_{i}}{m_{i}}
$$

Which can be written in terms of the $n_{l}$ not necessarily distinct networks intersected by initial sample (Tompson and Seber 1996).

$$
\hat{\mu}=\frac{1}{n_{1}} \sum_{i=1}^{n_{1}} \frac{1}{m_{1}} \sum_{j \in A_{i}} y_{j}=\frac{1}{n_{1}} \sum_{i=1}^{n_{1}} w_{i}=\bar{w}
$$

Where $w_{l}$ is the mean of the $m_{l}$ observation in $A_{l}$. This estimator has variance with unbiased estimator (Arabkhedri et al. 2010):

$$
\begin{aligned}
& \operatorname{Var}(\hat{\mu})=\frac{N-n_{1}}{N n_{1}(n-1)} \sum_{i=1}^{n_{1}}\left(w_{i}-\hat{\mu}\right)^{2} \\
& \mu=\frac{1}{N} \sum_{i=1}^{N} \frac{y_{i} I_{i}}{\pi_{i}} \\
& \mu=\text { Total of } y \text { values in the } i^{\text {th }} \text { network. }
\end{aligned}
$$

$I_{i}=1=$ If any quadrant in the $i^{t h}$ network, $0=$ otherwise
And $\pi_{i}=1-\left(\begin{array}{l}N-x_{i} \\ n\end{array}\right) /\left(\begin{array}{l}N \\ n\end{array}\right)$ is the probability that a quadrant in the $i^{\text {th }}$ containing $x_{i}$ quadrant is selected in the initial sample (Brown 1996):

$$
\begin{aligned}
& \pi_{i}=1-\left[\left(\begin{array}{l}
N-m_{i}-a \\
n_{i}
\end{array}\right) /\left(\begin{array}{l}
N \\
n_{1}
\end{array}\right)\right] \\
& \pi_{i}^{\prime}=1-\left[\left(\begin{array}{l}
N-m_{i} \\
n_{1}
\end{array}\right) /\left(\begin{array}{l}
N \\
n_{1}
\end{array}\right)\right] \\
& a_{k}=1-\left[\left(\begin{array}{l}
N-x_{k} \\
n_{1}
\end{array}\right) /\left(\begin{array}{l}
N \\
n_{1}
\end{array}\right)\right]
\end{aligned}
$$

In the following development, the total population size is equal to $N$ and the sampling frame contains $M$ clusters or primary sampling units. The number of elements or secondary sampling units in cluster $I$ is equal to $N I$, with $I$ $=1,2, \ldots, M$

Cluster size may or may not be constant across all $I$ (Brown 1999).

The sampling designs considered of ( $n=17)$ randomly selected positions for strips that were randomly arranged. We used a long-thin strip as a proxy for primary units which draws up along the width of the study area. The number of primary units for study sits was the same.

Simple random transect sampling calculated as follows which adopted from (Gruijter et al. 2005).

For simple random of $n$ transects the narrow strip estimator of the total is

$$
\hat{t}_{n s}(z)=\sum_{i=1}^{n} z_{i} \frac{B}{n 2 w}=\frac{B}{2 w} \bar{z}_{s}
$$

Where $z_{i}$ is the number of observed individuals in strip $i, B$ is the length of the baseline, $w$ is half the width of strips, and $\bar{z}_{s}$ is the sample mean of the numbers. (The term $\frac{B}{n 2 w}$ is the inverse of the transect inclusion probability density integrated over the width of the srtip). Then, the density is estimated by:

$$
\hat{\bar{z}}_{n s}=\frac{1}{A} \hat{t}_{n s}(z)
$$

Where $A$ is the (surface) area of the region

An alternative estimator, preferable in case of large differences in strip length, is the ratio estimator:

$$
\hat{\bar{z}}_{R}=\frac{\frac{B}{2 w} \sum_{i=1}^{n} z_{i}}{\sum_{i=1}^{n} L_{i}}
$$

The variance of it can be estimated by:

$$
\hat{V}\left(\hat{t}_{n s}(z)\right)=\left(\frac{B}{2 w}\right)^{2} \frac{\hat{S}^{2}\left(z_{i}\right)}{n}
$$


With $\hat{S}^{2}\left(z_{i}\right)$ the estimated spatial variance of the counts per strip:

$$
\hat{S}^{2}\left(z_{i}\right)=\frac{1}{n-1} \sum_{i=1^{\top}}^{n}\left(z_{i}-\bar{z}_{s}\right)^{2}
$$

The variance of the density can be estimated by dividing the estimated variance of the estimated total by $A^{2}$.

The narrow strip estimator is not entirely satisfying, because the detected individuals outside the narrow strip with perfect detectability are not used.

Ratio efficiency of ACS calculated as follows;

$R e=\frac{\operatorname{var}(\bar{y})}{\operatorname{var}(\hat{y})}$ sample variance of ACS var $(\hat{y}) ; \operatorname{Var}$ SRS

$\operatorname{var}(\bar{y})$. When re $\mathrm{S}>1 \mathrm{ACS}$ is more efficient RS.

\section{RESULTS AND DISCUSSION}

\section{Results}

To compare the performance of the proposed strip adaptive cluster sampling (SACS) it is necessary to know for each of the two inventory regions the variance of simple random sampling of the target object for both the adaptive and the non-adaptive approaches. The results for these approaches are illustrated in Tables 1 and 2 for two studied regions and are being used in this study to calculate the true variance of density estimates. Mean value of tree density estimates by SACS estimates was greater than SSD for all quadrat sizes except for quadrat size $15 \mathrm{~m}^{2}$ (Table 3 and 4). In general, with increasing the size of quadrat, mean of tree density not only increased but also percent of relative bias increased in comparison to SSD (Tables 1 and 2). This bias was in its upper level for $\mathrm{HH}$ estimator. The greatest and lowest relative bias of the mean tree density was recorded in quadrat size 25 and $15 \mathrm{~m}^{2}$ for HT estimators, whereas larger quadrat size/or length of strip results in decreasing the mean of tree density and it was close to full inventories mean's, which it was 0.3 for both of our studied areas. With increasing quadrat size from 15 to $25 \mathrm{~m}^{2}$ Coefficients of variation $(\mathrm{CV} \%)$ increased. The greatest increase in $\mathrm{CV} \%$ was related to $\mathrm{HH}$ than $\mathrm{HT}$ estimators in SACS compared to SSD approach (Table 4). Whereas variance of two estimators trends to decrease as quadrat size gets more.

Effects of quadrat size on statistical parameters showed that the small size of quadrat was produced less variability estimates compared to simple strip design (Tables 1 and 2). Comparison of two estimators showed that HT estimator was more efficient than $\mathrm{HH}$ in estimating mean, $\mathrm{CV} \%$ and variance (Var) compared to SSD approach. Estimated variance by HT estimator was smaller than one by the modified HH estimator (Tables 1 and 2). HT estimators had lower variability in estimating of mean, CV and Var. Overall, our results contributed to relative bias and percentage of relative bias showed that SACS was underestimated in estimating $\mathrm{CV} \%$ and Var, while it was overestimated only in estimating mean of tree density in all quadrate size except for size $15 \mathrm{~m}$ square in both sits compared to SSD procedure.

Table 1. Results of executed ASC approach for Namkhaneh management unit (initial sampling \#17)

\begin{tabular}{|c|c|c|c|c|c|c|c|c|c|c|c|c|c|}
\hline \multirow[t]{2}{*}{$\begin{array}{c}\text { quadrat size } \\
(\mathrm{m})\end{array}$} & \multirow[t]{2}{*}{$* \mathbf{N}$} & \multirow[t]{2}{*}{ Cluster } & \multirow[t]{2}{*}{ DFC } & \multicolumn{5}{|c|}{ HT estimator's } & \multicolumn{5}{|c|}{ HH estimator's } \\
\hline & & & & Mean & Var. & S.Er. & C.V\% & C.I. & Mean & Var. & S.Er. & C.V\% & C.I. \\
\hline 15 & 11398 & 42 & 5 & 0.5 & 0.054 & 0.23 & 46.475 & $0.34 \pm 0.460$ & 0.5 & 0.0045 & 0.067 & 13.416 & $0.05 \pm 0.134$ \\
\hline 20 & 6465 & 48 & 14 & 0.52 & 0.09 & 0.31 & 31.914 & $0.94 \pm 0.620$ & 0.52 & 0.011 & 0.104 & 9.894 & $1.06 \pm 0.208$ \\
\hline 25 & 4003 & 55 & 5 & 0.51 & 0.107 & 0.32 & 27.488 & $1.19 \pm 0.640$ & 0.51 & 0.0083 & 0.091 & 8.354 & $1.09 \pm 0.182$ \\
\hline
\end{tabular}

Note: $*=$ population size; $\mathrm{DFC}=$ define cluster

Table 2. Results of executed ASC approach for Patoom management unit (initial sampling \#17)

\begin{tabular}{|c|c|c|c|c|c|c|c|c|c|c|c|c|c|}
\hline \multirow{2}{*}{$\begin{array}{l}\text { Quadrat } \\
\text { size (m) }\end{array}$} & \multirow{2}{*}{$\mathbf{N}$} & \multirow{2}{*}{ Cluster } & \multirow{2}{*}{ DFC } & \multicolumn{5}{|c|}{ HT estimator's } & \multicolumn{5}{|c|}{ HH estimator's } \\
\hline & & & & Mean & Var. & S.Er. & C.V\% & C.I. & Mean & Var. & S.Er. & C.V\% & C.I. \\
\hline 15 & 6331 & 27 & 7 & 0.82 & 0.168 & 0.409 & 47.112 & $0.87 \pm 0.818$ & 0.91 & 0.088 & 0.296 & 30.582 & $0.97 \pm 0.593$ \\
\hline 20 & 3598 & 31 & 9 & 0.82 & 0.291 & 0.539 & 39.375 & $1.37 \pm 1.078$ & 0.9 & 0.17 & 0.412 & 30.316 & $1.36 \pm 0.825$ \\
\hline 25 & 2225 & 37 & 5 & 0.8 & 0.313 & 0.559 & 51.326 & $1.09 \pm 1.118$ & 0.92 & 0.21 & 0.458 & 42.431 & $1.08 \pm 0.917$ \\
\hline
\end{tabular}

Note: $*=$ population size; DFC $=$ define cluster

Table 3. Statistical calculations of simple strip inventory approach

\begin{tabular}{cllllllllll}
\hline \multirow{2}{*}{ Quadrat size (m) } & \multicolumn{9}{c}{ Namkhaneh } & \multicolumn{4}{c}{ Gorazbon } \\
\cline { 2 - 12 } & Mean & Var. & S.Er. & C.V\% & C.I. & Mean & Var. & S.Er. & C.V\% & C.I. \\
\hline 15 & 0.91 & 3.34 & 1.827 & 200.831 & $0.91 \pm 3.65$ & 0.82 & 3.13 & 1.769 & 198.784 & $0.89 \pm 3.538$ \\
20 & 0.92 & 1.2 & 1.095 & 120.378 & $0.91 \pm 2.190$ & 0.8 & 2.88 & 1.697 & 242.436 & $0.7 \pm 3.394$ \\
25 & 0.90 & 0.51 & 0.714 & 187.932 & $0.38 \pm 1.428$ & 0.81 & 1.44 & 1.200 & 272.727 & $0.44 \pm 2.400$ \\
\hline
\end{tabular}


Table 4. Results of bias and percent of bias in calculating statistical parameters of SACS approach

\begin{tabular}{lcllllllll}
\hline \multirow{2}{*}{ Variable } & \multirow{2}{*}{$\begin{array}{c}\text { Quadrat size } \\
\text { (m) }\end{array}$} & \multicolumn{4}{c}{ HT } & \multicolumn{3}{c}{ HH } & \multicolumn{3}{c}{ Gorazbon } & \multicolumn{2}{c}{ HH } \\
\cline { 3 - 10 } & & \multicolumn{1}{c}{ RB } & RB \% & \multicolumn{1}{c}{ RB } & RB \% & \multicolumn{1}{c}{ RB } & RB \% & RB & RB \% \\
\hline Mean & 15 & -0.41 & -45.054 & -0.41 & -45.054 & -0.02 & -2.24 & 0.08 & 8.99 \\
& 20 & 0.03 & 3.29 & 0.15 & 16.48 & 0.67 & 95.71 & 0.66 & 94.28 \\
& 25 & 0.81 & 213 & 0.17 & 186.84 & 0.65 & 147.72 & 0.64 & 145.45 \\
CV\% & 15 & -154.356 & -76.858 & -187.415 & -93.319 & -156.672 & -78.815 & -168.256 & -84.462 \\
& 20 & -88.464 & -73.488 & -110.484 & -91.78 & -203.061 & -83.758 & -212.120 & -87.495 \\
& 25 & -160.444 & -85.374 & -179.578 & -95.554 & -221.401 & -81.180 & -230.296 & -84.442 \\
Var & 15 & -3.286 & -98.383 & -3.335 & -99.865 & -2.962 & -94.632 & -3.042 & -97.188 \\
& 20 & -1.110 & -92.500 & -1.189 & -99.083 & -2.589 & -89.895 & 2.710 & -94.097 \\
& 25 & -0.403 & -79.019 & -0.501 & -98.372 & -1.127 & -78.263 & 1.230 & -85.416 \\
\hline
\end{tabular}

Note: $\mathrm{QS}=$ quadtat size in a contiguous transect, $\mathrm{RB}=$ relative bias, $\mathrm{RB} \%=$ percent of relative bias

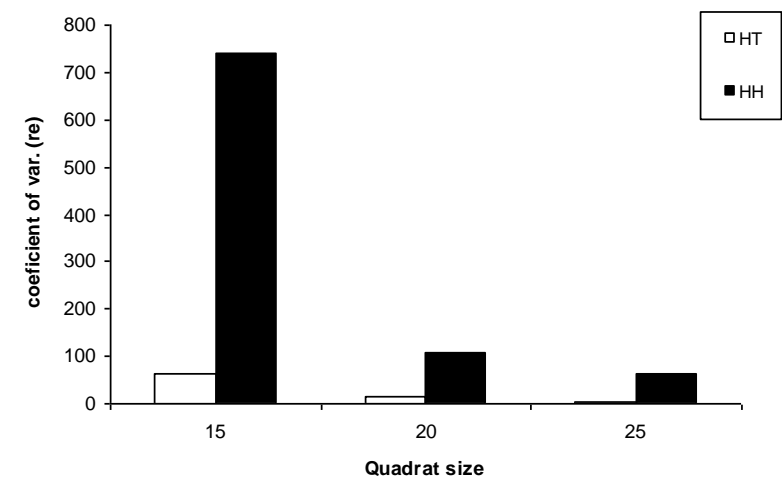

A

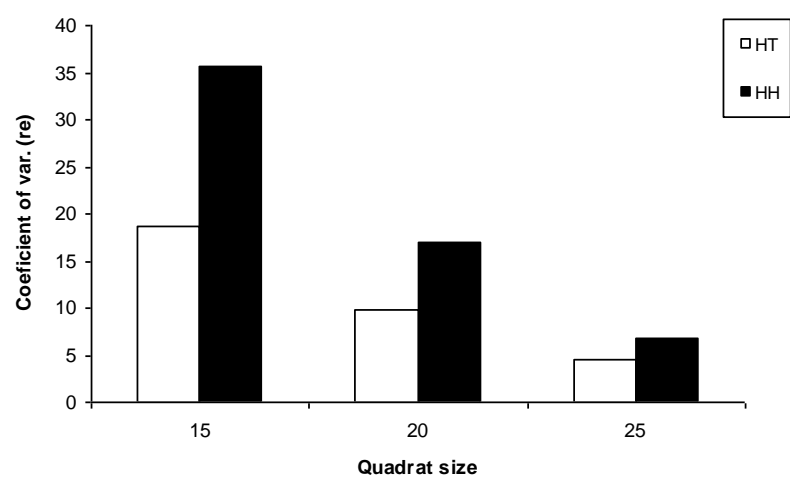

B

Figure 2. The efficiency of variances of SACS in Namkhane (A) and Patoom (B) district

\section{Discussion}

Due to the additional cost, the adaptive strategy should be saved for characteristics that are considered very important, and known to be both rare and found in clusters. The requirement that the trait motivating the adaptive strategy is being rare is one of partiality, not one of necessity from a statistical point of view. Results showed that if the number of strips (N) increases, the yield estimation of mean, variance coefficient and standard error for two estimators will be close to each other. This could explain this, the estimated probability of detecting a network is a function of the number of strips that intersect it, and the probability of selecting those transects with the initial survey design. Thus, large clusters have a higher probability of being detected, and are down-weighted in estimating of overall mean (Abrahamson et al. 2011). Small clusters and units that do not meet the criterion have smaller inclusion probabilities and contribute more to the overall mean. Our results are in line with the result of Conners and Schwager (2002).

In general, mean estimate of tree density by using proposed SACS approach was slightly greater than SSD. While it considerably produced close result to full inventory method. This ability will be more important in the future for forest inventory specialists that are looking for cost-effective techniques in rare species inventories.
Estimated variance by HT estimator was smaller than one by the modified $\mathrm{HH}$ estimator. These results are similar to the results of (Christman 1997) who concluded that Horwitz-Thompson estimator was less sensitive to the spatial distribution of population elements than the $\mathrm{HH}$ estimators, also they are more complex since they involve computing the inclusion probabilities of edge-units (Dryer 1999). Thompson and Seber (1996) and Christman (1997) compared the relative efficiencies of the two ACS estimation procedures (the HH and HT estimators). Both authors also note that the HT estimator has better efficiency - lower variance - than the $\mathrm{HH}$, when their differences increase so the initial sample size did too.

Larger quadrate sizes could enhance efficiency for some species, estimating tree density in quadrates less than $20 \mathrm{~m}$ square could increase measurement error or be difficult to implement where trees and other vegetation are abundant. At this research relative efficiency of adaptive cluster sampling is considerably affected by the relative sizes of the final and initial sample. The relationship between the efficiency of variance and the sample size had a power shape. Our results showed that the greatest efficiency of SACS was focused on small quadrate size. So with increasing quadrate size over $20 \mathrm{~m}^{2}$, the efficiency considerably decreased (Figure 2). We found that Selection of sampling strategies for forest rare species should be 
driven by specific research or sampling objectives. SACS shows good potential in this case-study in inventories of Wych elm in Hyrcanian forests. Future research may be required to broaden our view in other sampling design techniques like combination of SACS with two-phase sampling strategies in order to reduce sampling cost and improving its precision in field sampling of species which have cluster distribution pattern.

\section{REFERENCES}

Abella SR, Covington WW. 2004. Monitoring an Arizona ponderosa pine restoration: sampling efficiency and multivariate analysis of understory vegetation. Restor Ecol 12: 359-367.

Abrahamson I, Nelson CR, David L, Affleck R. 2011. Assessing the performance of sampling designs for measuring the abundance of understory plants. Ecol Appl 21 (2): 452-464.

Acharya B, Bhattarai G, Gier AD, Stein A. 2000. Systematic adaptive cluster sampling for the assessment of rare tree species in Nepal. For Ecol Manag 137: 65-73.

Arabkhedri M, Lai FS, Noor Akma I, Mohamad-Roslan MK. 2010. Adaptive cluster sampling for a temporal-scale population. Malaysian J Math Sci 4 (1): 53-73.

Barbier S. 2008. Influence of tree species on understory vegetation diversity and mechanisms involved: a critical review for temperate and boreal forests. For Ecol and Manag 254: 1-15.

Barlow J. 1995. The abundance of cetaceans in California waters. 1. Ship surveys in summer and fall of 1991. Fish Bull 93: 1-14.

Brown JA. 1996. The relative efficiency of adaptive cluster sampling for ecological surveys. Math Inform Sci Rep, ser B: 96-108.

Brown JA. 1999. A comparison of two adaptive sampling designs. Austr N Z J Stat 41 (4): 395-403.

Christman MC. 1997. Efficiency of some sampling designs for spatially clustered populations. Environmetrics 8 (2): 145-166.
Conners ME, Schwager SJ. 2002. The use of adaptive cluster sampling for hydroacoustic surveys. ICES J Mar Sci 59: 1314-1325.

Dryer DC. 1999. Getting personal with computers: How to design personalities for agents. Appl Artif Intell 13: 273-295.

Ensign WE, Angermeier Pl, Dolloff CA. 1995. Use of line- transect methods to estimate abundance of benthic stream shes. Canadian $\mathrm{J}$ Fish Aquat Sci 52: 213-222.

Evans AM, Gregoire TG. 2006. The tree crown distribution of hemlock woolly adelgid, Adelges tsugae (Hem., Adelgidae) from randomized branch sampling. J Appl Entomol 131: 26-33.

Goslee SC. 2006. Behavior of vegetation sampling methods in the presence of spatial autocorrelation. Plant Ecol 187 (2): 203-212.

Greig-Smith P. 1983. Quantitative plant ecology. Blackwell Scientific Publications. Oxford.

Gruijter JD, Brus D, Bierkens M, Knotters M. 2005. Sampling for Natural Resource Monitoring. Springer, Berlin.

Limgta T, Fries C. 1995. An integrated forest inventory in a managed north Swedish forest landscape for estimating growing stock and coarse woody debris. In: Kohl M, Bachmann P, Brassel P, Preto G (eds). Proceedings, The Monte Veritt conference on forest survey designs. 1994 May 2-7, Monte Verit/l, Switzerland, Swiss Federal Institute for Forest, Snow and Landscape Research, Binnensdorf.

Pontius JA. 1997. Strip adaptive cluster sampling: Probability proportional to size selection of primary units. Biometrics 53 (3): 1092-1096.

Sabeti H. 1976. Trees and shrubs of Iran. Publication of Yazd University. Iran.

Salehi M, Seber GA. 1997. Two-stage adaptive cluster sampling. Biometrics 53: 959-970.

Stohlgren TJ, Falkner MB, Schell LD. 1995. A modified-Whittaker nested vegetation sampling method. Vegetatio 117: 113-121.

Stohlgren, TJ. 2007. Measuring Plant Diversity: Lessons from the Field. Oxford University Press, New York.

Tompson SK, Seber GAF. 1996. Adaptive Sampling. John Wiley \& Sons, Inc., New York.

Tompson SK. 1990. Adaptive cluster sampling. J Amer Stat Assoc 85: 1050-1059

Tompson SK. 1992. Sampling. John Wiley \& Sons, Inc., New York. 